\title{
What is the phylogenetic position of Cryptosporidium?
}

\author{
Guan Zhu, ${ }^{1}$ Janet S. Keithly ${ }^{1}$ and Herve Philippe ${ }^{2}$ \\ Author for correspondence: Janet S. Keithly. Tel: +1 518473 2692. Fax: +1 5184736150 . \\ e-mail: keithly@wadsworth.org
}

\footnotetext{
1 Wadsworth Center, New York State Department of Health, David Axelrod Institute for Public Health, 120 New Scotland Avenue, Albany, NY 12201-2002, USA

2 University of Paris-South, Orsay, France
}

\begin{abstract}
The phylogenetic position of Cryptosporidium is elusive. Although previous studies based solely upon small-subunit (SSU) rRNA sequences suggested that the genus was an early emerging lineage among the Apicomplexa, bootstrap support for this placement was low. Here, the phylogenetic position of Cryptosporidium has been re-evaluated for SSU rRNA, fused SSU/large-subunit (LSU) rRNA and six protein sequences using traditional distance-based neighbour-joining, maximum-parsimony and maximum-likelihood methods of phylogenetic reconstruction as well as the new Slow-Fast analysis, which focuses upon the slowly evolving positions within sequences and is especially useful if a long-branch attraction (LBA) artefact is suspected. All the methods of reconstruction indicated a trend for the early emergence of Cryptosporidium at the base of the Apicomplexa and showed that an LBA artefact plays no role in this placement. Although the inclusion of additional numbers of neither species nor genes has significantly enhanced the bootstrap support for this phylogenetic position, recent biochemical, molecular and ultrastructural data are congruent with it. Therefore, we favour a working hypothesis that this genus constitutes an early emerging branch of the Apicomplexa.
\end{abstract}

Keywords: Apicomplexa, Coccidia, Cryptosporidium parvum, molecular phylogeny, rRNA

\section{INTRODUCTION}

Cryptosporidium parvum is an opportunistic pathogen that causes life-threatening diarrhoea in AIDS patients. Although C. parvum is a typical coccidium in its morphology (apical complex, dense bodies, micronemes, rhoptries) and lifestyle (Fayer et al., 1997), there are some fundamental differences between this parasitic protist and the eucoccidia. These include: (i) the extracytoplasmic but intracellular location of $C$. parvum in a parasitophorous vacuole just beneath the enterocyte apical membrane (Fayer et al., 1997); (ii) the presence of an acristate, ribosome-studded mitochondrion posterior to the nucleus (Riordan et al., 1999); (iii) plant-like polyamine biosynthesis by decarboxylation of arginine rather than ornithine

Abbreviations: BP, bootstrap proportion; LBA, long-branch attraction; LSU, large-subunit; ML, maximum-likelihood; MP, maximum-parsimony; NJ, neighbour-joining; SSU, small-subunit.

The GenBank accession number for the sequence of Cryptosporidium parvum rDNA clone CprRNA-KSUH4 is AF040725.
(Keithly et al., 1997); (iv) the apparent lack of a plastid or its genome (Zhu et al., 2000); (v) insensitivity to most anticoccidial drugs (Coombs et al., 1997; Coombs, 1999; Woods et al., 1996); and (vi) sporulation of oocysts within the intestine resulting in enterocyte reinvasion and prolonged, life-threatening infection in immunocompromised patients (Fayer et al., 1997).

Phylogenetic analyses using small-subunit (SSU) rRNA sequences have indicated that the genus Cryptosporidium does not form a monophyletic clade with either intestinal (Eimeria, Isospora) or cyst-forming (Sarcocystis, Toxoplasma) eucoccidia and that the genus is instead a sister-group of the Coccidia+ Haematozoa (Morrison \& Ellis, 1997; Escalante \& Ayala, 1995). In fact, the class Coccidia was shown to be monophyletic only if the genus Cryptosporidium was excluded from the analysis (Morrison \& Ellis, 1997), and the genus could even be used as an outgroup to determine the phylogenetic position of eucoccidia (Barta et al., 1997). Although these studies suggest that Cryptosporidium may be an early emerging lineage 
among the apicomplexans, bootstrap support for this placement has been low (Escalante \& Ayala, 1995; Gajadhar et al., 1991; Van de Peer \& De Wachter, 1997).

There are a number of possible reasons why the previous data from SSU rRNA might be misleading: (i) early emerging lineages are often fast-evolving and can be misplaced due to a long-branch attraction (LBA) artefact (Philippe \& Laurent, 1998); (ii) the use of inadequate methods of tree reconstruction (Yang, 1996); (iii) mutational saturation of sites, e.g. if outgroups are too distant from ingroup species (Philippe \& Adoutte, 1998); (iv) lack of diversity of species in the database for the genes being analysed (Lecointre et al., 1993; Graybeal, 1998); and (v) exclusive reliance upon a single gene (SSU rRNA) dataset (Escalante \& Ayala, 1995; Gajadhar et al., 1991). Therefore, in this paper, we present a detailed phylogenetic analysis of the SSU and fused largesubunit (LSU)/SSU rRNA, as well as six proteinencoding genes in order to re-evaluate the phylogenetic position of Cryptosporidium. Analyses included traditional distance-based neighbour-joining (NJ) (Saitou \& Nei, 1987), maximum-parsimony (MP) and maximum-likelihood (ML) methods of phylogenetic construction (Strimmer \& von Haeseler, 1996; Swofford, 1993), as well as a new Slow-Fast (S-F) analysis (Brinkmann \& Philippe, 1999; Lopez et al., 1999), which focuses upon the slowly evolving positions and is especially helpful if misplacement due to an LBA artefact is suspected. All methods of reconstruction using these three datasets indicated a trend for the early emergence of Cryptosporidium, still with only low to moderate support, however, and all indicated that LBA plays no role in this placement.

\section{METHODS}

Molecular cloning and sequencing. Free sporozoites of $C$. parvum [Kansas State University strain (KSU-1)] were obtained by excystation and genomic DNA was isolated using a Puregene kit (Gentra Systems) as described previously (Zhu \& Keithly, 1997). A fragment of the SSU rRNA gene was first amplified from genomic DNA by PCR using two primers whose sequence is conserved in most eukaryotic cells, CRib-10F (5'-GTTGATCCTGCCAGTAGTCATATGC-3') and CRib-10R (5'-GTTCACCTACGGAAACCTTGTTACG-3'). This amplicon was then used to screen a pBluescript SK $(+)$ HindIII genomic DNA library. Of several positive clones containing SSU and LSU rRNA genes, both strands of one (CprRNA-KSUH4; accession no. AF040725) were sequenced completely by us and then used for alignments and phylogenetic analyses. Oligonucleotide synthesis and automated DNA sequencing were provided by the Wadsworth Center Molecular Genetics Core.

Phylogenetic analyses of rRNA and proteins. Aligned sequences of rRNA from members of the Alveolata were retrieved from the rRNA database at http://rrna.uia.ac.be/ (Van de Peer et al., 1999). Partial or redundant sequences, as well as those with obvious sequencing errors, were discarded, yielding a dataset of 83 SSU and 19 LSU rRNA genes. All sequences homologous to $\alpha$ - and $\beta$-tubulin, cyclin-dependent protein kinase (cdc2), dihydrofolate reductase-thymidylate synthase (DHFR-TS), cytosolic $70 \mathrm{kDa}$ heat-shock protein (HSP70) and elongation factor EF-1 $\alpha$ were identified by a BLAST search (blast@ncbi.nlm.nih.gov) and were retrieved automatically with the programs BLAST2RETP and RETP2ALI (P. Lopez, personal communication). Alignments were performed visually with the help of the ED program version 1.0 (Philippe, 1993).

Phylogenetic trees were constructed by ML, MP and NJ methods using the programs PROTML version 2.3 (Adachi \& Hasegawa, 1996), PUZZLE version 4.01 (Strimmer \& von Haeseler, 1996), PAup version 3.1.1 (Swofford, 1993) and NJ version 1.0 (Philippe, 1993). Nucleotide and protein distances were computed with the substitution models of Tamura \& Nei (1993) and Kimura (1980), respectively. MP trees were obtained by 10 random addition heuristic search replicates. ML trees were obtained by the quick-add operational taxonomic units (OTU) search of the NUCML/ PROTML programs (Kishino et al., 1990), using the Jones, Taylor and Thornton (JTT) model of amino acid substitution (Jones et al., 1992), in which the 2000 top-ranking trees (options $-j f-q-n$ 2000) were retained. These were evaluated as users' trees with the PUZZLE program, the model of rate heterogeneity being gamma-distributed into eight rate categories. Best trees obtained with PROTML were identical to the best trees obtained by PUZZLE (data not shown). Bootstrap proportions (BP) (Felsenstein, 1985) were calculated by the analysis of 100 (1000) replicates for MP (NJ) trees, whereas those computed for ML used the resampling estimated log-likelihood (RELL) method of the NUCML/PROTML program (Kishino et al., 1990). The $\alpha$ parameter of the gamma distribution was estimated using the phylogenetic analysis by maximum-likelihood (PAML) program (Yang, 1997).

To test for potential reconstruction errors due to an LBA artefact, the S-F method (Brinkmann \& Philippe, 1999) was used in an analysis of the complete SSU rRNA dataset, which included eight monophyletic groups: Ciliophora, Dinozoa, Cryptosporidium, Eimeria and relatives, Sarcocystis and relatives, Theileria and relatives, Babesia and Plasmodium. This method permits the evolution of relationships to be studied as more and more variable positions are added to the matrix, starting with the most slowly evolving positions. The evolutionary rate of each position was evaluated as the total number of steps in the most parsimonious tree for each monophyletic group. To reduce potential reconstruction errors due to LBA artefact further, this method was also applied after removing the groups displaying long branches: (i) Plasmodium group; (ii) Plasmodium and Eimeria groups; and (iii) ciliates plus the Plasmodium and Eimeria groups.

\section{RESULTS}

Our SSU and fused LSU/SSU rRNA data confirm that Cryptosporidium belongs to the Apicomplexa and does not form a monophyletic clade with any of the eucoccidia (Figs 1 and 2) and that there is a tendency for its early emergence at the base of the Apicomplexa.

\section{SSU rRNA analyses}

It is well known that phylogenetic reconstruction can be improved by adding diverse species to reduce errors introduced by LBA artefacts (Hendy \& Penny, 


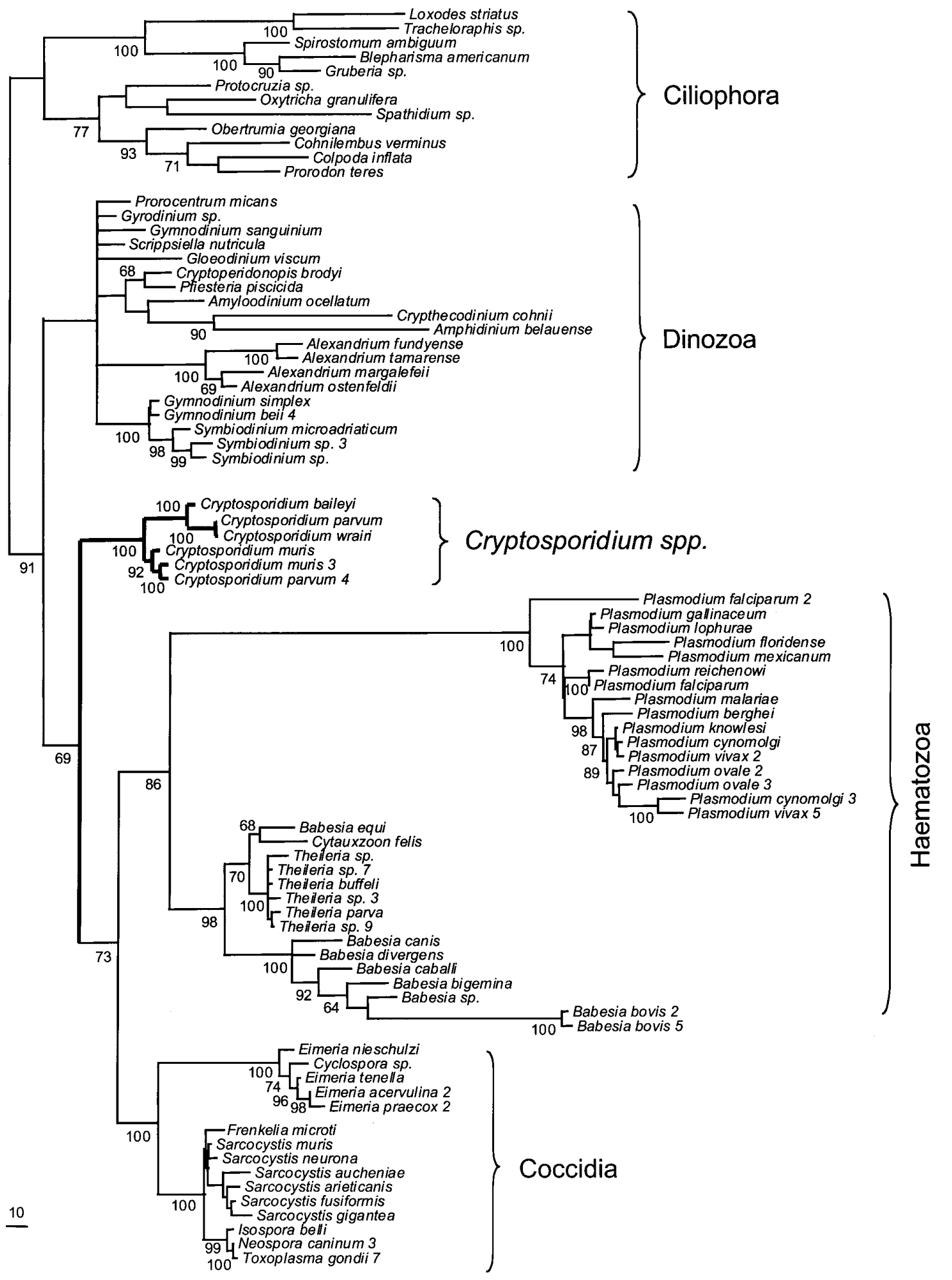

Fig. 1. MP tree inferred from an SSU rRNA dataset containing 52 complete sequences within the phylum Apicomplexa using 12 Ciliophora and 19 Dinozoa as outgroups to root the tree. All are members of the Alveolata. The BP is $73 \%$ for the early emergence of Cryptosporidium and this node is also supported by a Bremer decay index (Bremer, 1988) of 2.

1989; Lecointre et al., 1993; Graybeal, 1998; but see Kim, 1996; Poe \& Swofford, 1999). Although there are about 150 sequences of Apicomplexa available, we have selected only those 52 SSU rRNA sequences that are complete and that represent a reasonable diversity of genera within the phylum Apicomplexa (Fig. 1). 


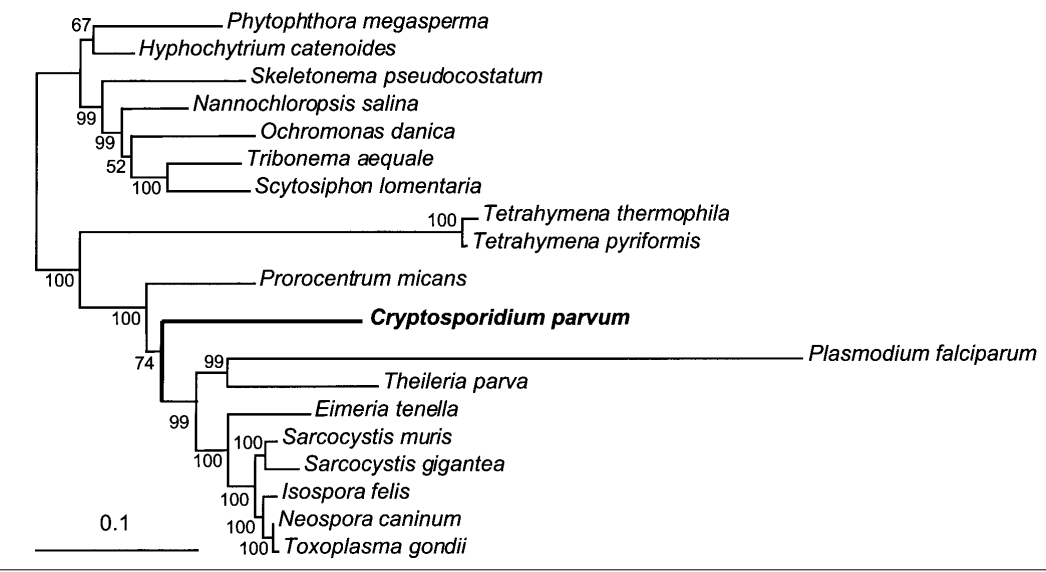

\begin{abstract}
Fig. 2. ML tree inferred from fused SSU and LSU rRNA genes. BPs at each node were estimated using the RELL method as implemented in the NUCML/PROTML program (Kishino et al., 1990). The $\alpha$-parameter of the gamma distribution was estimated with the PAML programs (Yang, 1997). In this phylogenetic reconstruction, there is also a trend for the emergence of Cryptosporidium at the base of the Apicomplexa (BP $=99 \%$ ).
\end{abstract}

Species from more divergent genera, e.g. Caryospora, Hepatozoon, Lankesterella and Perkinsus, have not been included because too few or only partial sequences are available. Furthermore, outgroup sequences from 19 Dinozoa and 12 Ciliophora (Fig. 1) were selected from within the Alveolata to minimize errors due to mutational saturation (Philippe \& Adoutte, 1998), which can bias reconstructions if the outgroup is too distant from species within the ingroup. Although both MP and NJ methods of reconstruction were used, only MP is shown (Fig. 1). Our data clearly show that there is moderate support for the early emergence of Cryptosporidium (Fig. 1; BP $=73 \%$ ), i.e. that the genus is a sister-group of the [Coccidia + Haematozoa] rather than a member of the class Coccidia (Morrison \& Ellis, 1997; Escalante \& Ayala, 1995).

Because among-site rate variation can also influence tree reconstruction (Fitch, 1971; Hendy \& Penny, 1989; Van de Peer \& De Wachter, 1997), the degree to which this occurs was measured using the PAML program and the $\alpha$-parameter of the gamma law was estimated to be 0.325 . This important heterogeneity has been taken into account in the construction of the NJ tree using the method of Tamura \& Nei (1993). The results were virtually identical to the MP tree, in which among-site variation was ignored. To verify further that the fast-evolving positions were not misleading, the SSU rRNAs of these genera were analysed using the S-F method (Brinkmann \& Philippe, 1999). This allows an objective selection of slowly evolving positions and reduces the noise encountered by fastevolving ones and is especially helpful to test for LBA artefact. Analysis by S-F confirmed that Plasmodium evolves significantly faster than other apicomplexans (Fig. 1) and that the long branch observed in Fig. 1 is real. Thus, these additional SSU rRNA analyses by the S-F method suggest that the early emergence of Cryptosporidium is not due to an LBA artefact, but to a few, very real, slowly evolving positions within the SSU rRNA of this genus. For example, when positions with fewer than two changes are analysed, this node is supported by a Bremer decay index (Bremer, 1988) of
2, which again favours the trend toward an early emergence of Cryptosporidium.

\section{Fused LSU/SSUrRNA analyses}

Although there are thousands of SSU rRNA sequences for diverse species in the molecular database, it contains only a limited number of complete (or nearly complete) LSU rRNA sequences. Therefore, outgroups for these analyses included seven stramenopiles, two ciliates and one dinoflagellate (Fig. 2). Unlike the $>150$ SSU rRNA sequences for apicomplexans, the LSU sequences of only eight species are available (Gagnon et al., 1996; Rogers et al., 1996; Mugridge et al., 1999). Here, we have added the complete sequence of $C$. parvum rDNA (AF040725). An analysis of Cryptosporidium fused LSU/SSU rRNA sequences generally agrees with those for SSU rRNA alone and the results are congruent with each other regardless of the method of phylogenetic reconstruction. Only the ML tree is shown (Fig. 2). The $\mathrm{NJ}$ and MP trees were almost identical to the ML one: the only differences were that Phytophthora emerged earliest within the Stramenopila and that $C$. parvum was the sister-group of Plasmodium, respectively. These LSU/SSU rRNA-inferred phylogenies are also congruent with current taxonomy (Gajadhar et al., 1991; Van de Peer \& De Wachter, 1997; Van der Auwera \& De Wachter, 1998) in that the Dinozoa (Prorocentrum) are a sister-group of the Apicomplexa and that both of these alveolates are closely related to the Ciliophora (Tetrahymena). The phylogeny within the Stramenopila, using 19 species instead of eight, was reaffirmed (Van der Auwera \& De Wachter, 1998). As expected, within the phylum Apicomplexa the monophyly of the Coccidia (sensu stricto) and Haematozoa (Fig. 2; $\mathrm{BP}=99 \%$ ) was confirmed in all trees. Somewhat unexpectedly, however, Cryptosporidium emerged in two quite different phylogenetic positions: (i) at the base of the Apicomplexa by the ML (or MP) method (Fig. 2; $\mathrm{BP}=99 \%$ ) or (ii) as a sister-group to Plasmodium by the NJ method ( $\mathrm{BP}=60 \%$; data not shown).

These two positions for Cryptosporidium are signifi- 
cantly different, as revealed both by the high $\mathrm{BP}$ value for the ML reconstruction (Fig. 2; BP $=99 \%$ ) and by a Kishino-Hasegawa test (data not shown). Furthermore, the discrepancy between the ML and NJ methods is not due to among-site rate variation, because both methods assume rate heterogeneity using the gamma law, i.e. PUZZLE (Strimmer \& von Haeseler, 1996) and Tamura \& Nei (1993), respectively. Moreover, the same model of nucleotide substitutions was assumed. Therefore, the discrepancy could be due to different sensitivities of the methods used for tree reconstruction to model violations.

We have detected at least two serious model violations: (i) significantly heterogeneous nucleotide compositions between the stramenopiles Scytosiphon and Tribonema (GC-rich) and the alveolates Cryptosporidium and Plasmodium (AT-rich) and (ii) significantly heterogeneous evolutionary rates of the positions, according to the covarion model. Covarions are the sets of positions that are free to vary (Fitch \& Markowitz, 1970) and they can change throughout evolutionary time (Fitch, 1971; Lockhart et al., 1996; Lopez et al., 1999). This implies that a position can be constant in one taxon and yet highly variable in another. We tested the heterogeneity of evolutionary rate for each position using the method of Lopez et al. (1999). This analysis showed that the SSU rRNA was significantly heterogeneous $(1 \%$ level $)$ and that this was not due to any particular group, since identical results were obtained even when each group was removed separately from the analysis. Because the ML method (Kishino et al., 1990) is more robust against model violations than the NJ method (Saitou \& Nei, 1987), the placement of Cryptosporidium at the base of the Apicomplexa $(\mathrm{BP}=99 \%)$ is also the preferred phylogenetic position based upon LSU/SSU rRNA reconstructions and is therefore congruent with the data for SSU rRNA.

As mentioned previously, topological agreement between trees reconstructed from divergently evolving genes can provide an additional test to corroborate or refute those data derived from rRNA gene databases (Miyamoto \& Fitch, 1995). Therefore, six protein datasets for which several apicomplexan sequences are available were examined to see whether they also supported the early emergence of Cryptosporidium.

\section{Protein phylogenetic analyses}

Phylogenetic reconstructions for $\alpha$-tubulin, $\beta$-tubulin, EF-1 $\alpha$, HSP70, cdc2 and DHFR-TS (Fig. 3) used the Euglenozoa, Stramenopila and/or Ciliophora as outgroups, again to minimize bias due to mutational saturation (Philippe \& Adoutte, 1998). As mentioned before, trees inferred by the ML method are most robust against model violations; these are therefore depicted in Fig. 3. Reconstructions for these six proteins are ordered from those most congruent with the rRNA trees (Fig. 3a-b) to those least congruent (Fig. 3e-f). For clarity, these ML trees are first described excluding the genus Cryptosporidium, since the phylogenetic positions within the Apicomplexa are congruent with placement based upon SSU rRNA, i.e. (Toxoplasma, Eimeria), [(Babesia, Theileria), Plasmodium]. Although the Apicomplexa and/or the Ciliophora were not always monophyletic in these analyses, differences usually occurred only at non-robust nodes. This phenomenon is especially well-documented for EF-1 $\alpha$ (Moreira et al., 1999).

Unlike the basically congruent phylogenetic picture for the six proteins among the Alveolata and the Apicomplexa when the genus Cryptosporidium was excluded, there was no consensus when this genus was considered (Fig. 3a-f). However, there was a trend that favoured the early emergence of Cryptosporidium, but again with only low $(\mathrm{BP}=45-64 \%)$ to moderate (BP $=70-85 \%$ ) support. Specifically, the phylogenetic reconstruction using $\beta$-tubulin and DHFR-TS (Fig. $3 \mathrm{a}-\mathrm{b})$ placed Cryptosporidium at the base of the Apicomplexa $(\mathrm{BP}=70-85 \%)$, agreeing with its placement when ML/MP methods were used for SSU rRNA (Fig. 1). On the other hand, when $\alpha$-tubulin and HSP70 were analysed (Fig. 3c-d), Cryptosporidium emerged as a sister-group to Plasmodium ( $\mathrm{BP}=79 \%$ and $64 \%$, respectively), agreeing with the NJ method of reconstruction for SSU rRNA (data not shown). Finally, the least congruent phylogenetic placements of Cryptosporidium were those for EF- $1 \alpha$ (Fig. 3e) and cdc2 (Fig. 3f). In the former, this genus emerged as a sister-group to the cyst-forming eucoccidium Toxoplasma $(\mathrm{BP}=45 \%)$ and, in the latter, the genus emerged prior to the ciliate Paramecium $(\mathrm{BP}=49 \%)$, thus rendering the Apicomplexa paraphyletic (Fig. 3f).

Although the reconstruction methods for SSU rRNA, LSU/SSU rRNA and six proteins all indicated a tendency for the early emergence of Cryptosporidium, which is a hypothesis favoured by us (and others), the inclusion of additional numbers of neither species nor genes has enhanced significantly the bootstrap support for this placement.

\section{DISCUSSION}

What, if anything, can be inferred from these rather diverse data, and why do discrepancies still occur? As mentioned previously, there may be several reasons for the contradictions among these datasets: (i) limitations due to tree reconstruction artefacts; (ii) too short and/or too limited diversity of dataset sequences; and (iii) genuine differences in the genome and/or biology of the genus from its nearest relatives. Firstly, although our analyses indicate that the early emergence of Cryptosporidium is not due to an LBA artefact, other model violations may occur. We have shown, for example, that SSU rRNA sequences fit significantly better to a covarion model than to a rate-across-sites model. This important model violation can bias even the most elaborate methods, including that of ML with a gamma law and the Tamura/Nei model implemented in PUZZLE (Strimmer \& von Haeseler, 1996), suggesting 
(a) $\beta$-Tubulin

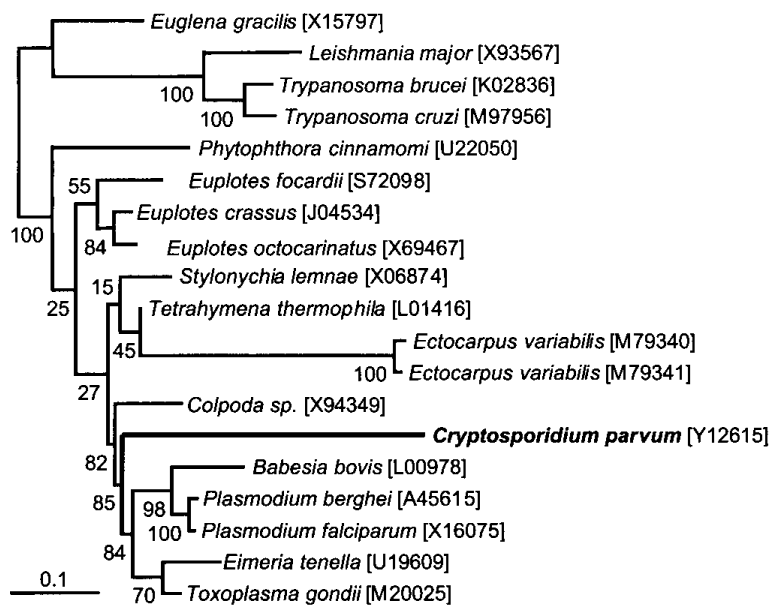

(b) DHFR

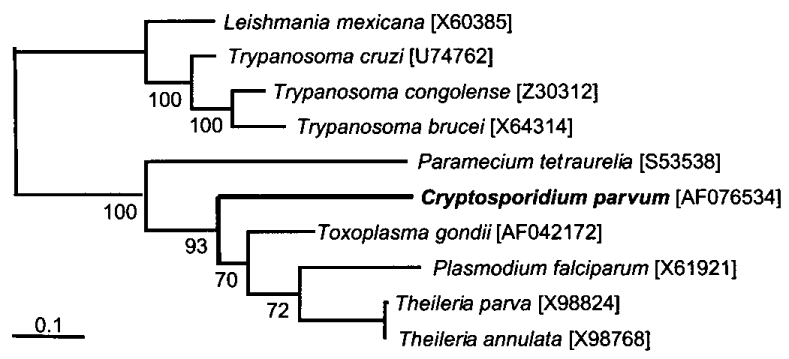

(c) $\alpha$-Tubulin

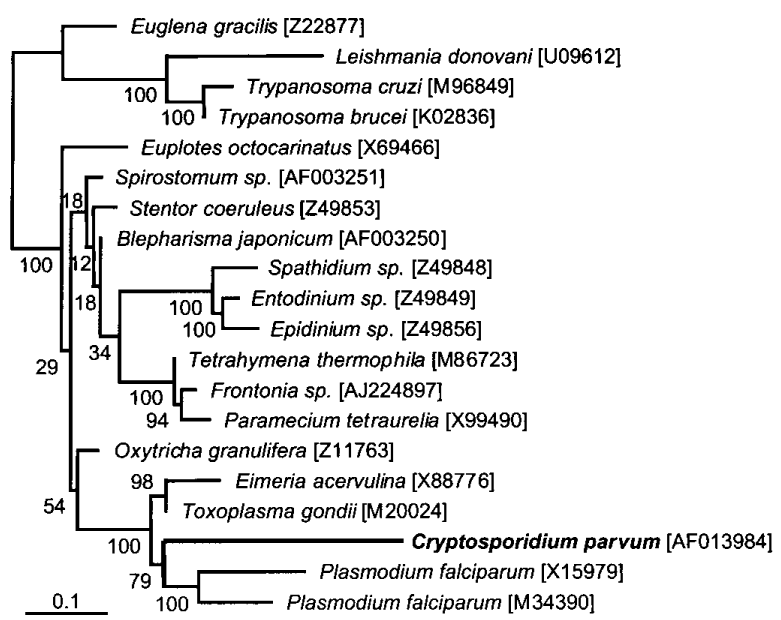

(d) HSP70

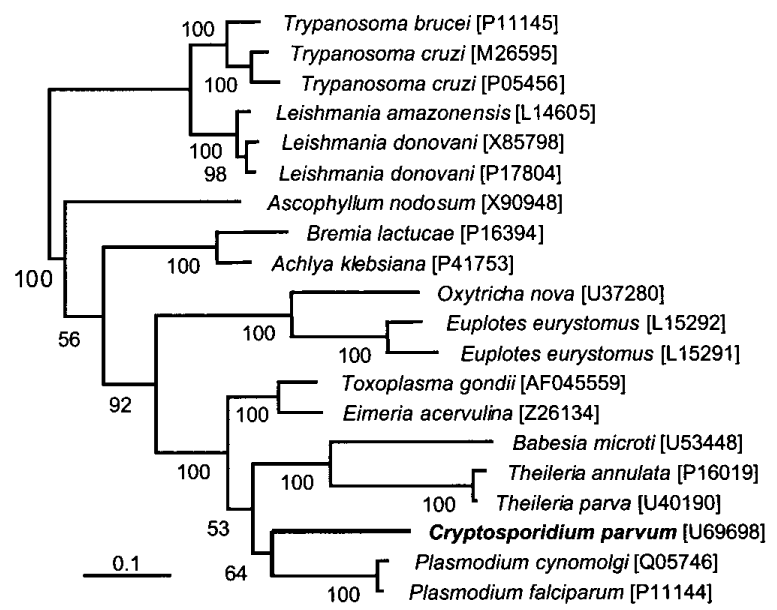

(e) EF-1 $\alpha$

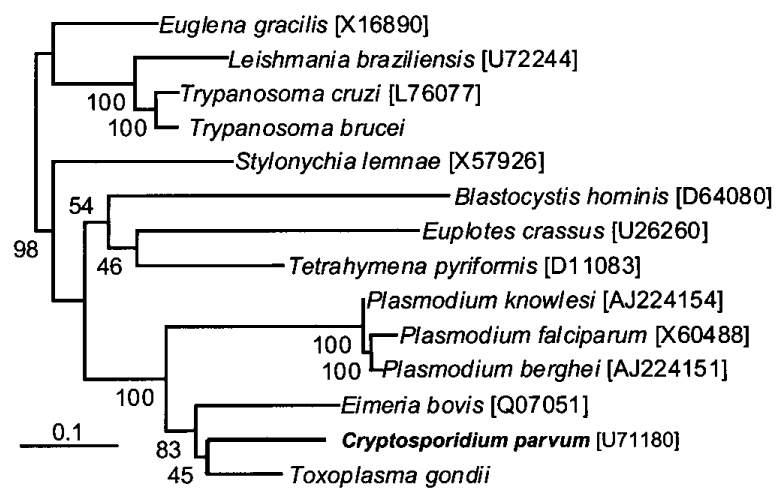

(f) $\operatorname{cdc} 2$

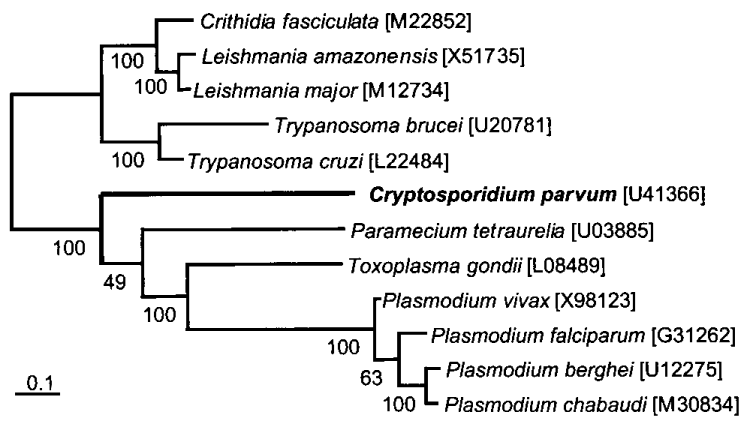

Fig. 3. $M L$ phylogenetic trees of six different proteins using the Euglenozoa, Stramenopila and/or Ciliophora as outgroups: (a) $\beta$-tubulin, (b) DHFR-TS, (c) $\alpha$-tubulin, (d) HSP70, (e) EF-1 $\alpha$ and (f) cdc2. The phylogenetic position of Cryptosporidium varies depending upon the protein examined: $(a, b)$ at the base of the Apicomplexa, in agreement with ML (MP) rRNA; (c, d) as a sister-group to Plasmodium, agreeing with NJ rRNA; (e) as a sister-group to the eucoccidium Toxoplasma; and (f) prior to the ciliates, rendering the Apicomplexa paraphyletic. 
that one must be cautious when interpreting these results. Moreover, another model violation, heterogeneity in nucleotide composition, has been demonstrated by us. Therefore, although we have attempted to limit errors due to tree reconstruction artefacts, it is clear that some must still exist and that these cannot be resolved easily with the phylogenetic analytical methods currently available to us.

Secondly, it is possible that there are simply too few diverse species available in the database to determine LSU rRNA (Fig. 2) and some protein phylogenies (Fig. 3) among the Alveolata and the Apicomplexa. Whether the phylogenetic position of Cryptosporidium is truly ancestral to the Apicomplexa or its characters are derived following a loss of genes, organelles and/or functions is difficult to determine with so little information available for the outgroups most likely to shed light on this problem, i.e. Dinozoa, Ciliophora, Stramenopila. It is further compounded by the paucity of diverse rRNA and protein sequences in the class Haematozoa, the absence of these sequences in the class Gregarina, order Eugregarina, within the phylum Apicomplexa, and the uncertain phylogenetic position of the genus Perkinsus (Goggin \& Barker, 1993; Siddall et al., 1997). Furthermore, it is well known that gene trees can differ markedly from species trees because of gene duplication, gene loss and/or horizontal gene transfer (Maddison, 1997). We know that this problem exists within the phylum Apicomplexa because there are at least two genes for $\alpha$-tubulin (Philippe \& Adoutte, 1998). Although it is currently difficult to resolve these problems, the genome projects for apicomplexans, dinoflagellates and ciliates should continue to provide valuable information.

These phylogenetic data, indicating the possible early emergence of Cryptosporidium, are also correlated with recent structural, biochemical and molecular studies of this parasite. Although the life cycle of $C$. parvum is typically coccidian, i.e. one or more stages of merogony, a sexual stage producing oocysts and sporulation of oocysts to form sporozoites, it differs from typical eucoccidia both in its intracellular location just beneath the plasma membrane of the enterocyte and sporulation within the gut rather than in the environment (Fayer et al., 1997). All eucoccidia and haematozoa studied to date possess both a plastid organelle (apicoplast) and genome (Kohler et al., 1997; Lang-Unnasch et al., 1998; Wilson \& Williamson, 1997) and elongate mitochondria with cristae in which genes are encoded (Wilson \& Williamson, 1997). Unlike these near relatives, $C$. parvum appears to lack both a plastid and its genome (Zhu et al., 2000), but has an unusual, ribosomestudded mitochondrion that is acristate and lies posterior to the nucleus (Riordan et al., 1999). In addition, metabolic peculiarities occur that distinguish this group from its nearest relatives (Coombs et al., 1997; Denton et al., 1996; Fayer et al., 1997; Keithly et $a l ., 1997)$. Unlike the eucoccidia and haematozoa, $C$. parvum has a plant-type polyamine biosynthetic path- way that permits decarboxylation of arginine rather than ornithine to form putrescine (Keithly et al., 1997). Like its coccidian relatives Toxoplasma gondii and Eimeria tenella, glycolysis in C. parvum appears to be mainly fermentative but, unlike them, one of the major enzymes in this pathway, pyruvate kinase, is unregulated (Denton et al., 1996). Furthermore, most anticoccidial drugs that effectively inhibit the growth of the intestinal eucoccidia Eimeria and Isospora in vitro and in vivo have similar $\mathrm{ED}_{50}$ for growth inhibition of the cyst-forming eucoccidia Toxoplasma and Sarcocystis (Coombs, 1999; Coombs et al., 1997; Woods et al., 1996). Yet, in spite of repeated attempts by a variety of laboratories, most of these inhibitors have little or no effect against growth of Cryptosporidium in vitro or in vivo (Coombs, 1999; Woods et al., 1996). Similarly, drugs effective against species of Plasmodium and Babesia have little effect against cryptosporidia (Woods et al., 1996), again suggesting fundamental biological differences between these genera comprising the classes Coccidia and Haematozoa and the genus Cryptosporidium.

Although these biochemical, drug-testing, molecular, phylogenetic and ultrastructural data are consistent with the emergence of Cryptosporidium at the base of the Apicomplexa, it is premature to state this categorically. Perhaps, then, a useful working hypothesis, and one that we favour, would be that this genus appears to be an early emerging branch of the Apicomplexa. If the early emergence of Cryptosporidium can be confirmed, the similarities observed between this genus and the Coccidia would suggest that they are ancestral and that the Haematozoa are derived from a coccidian-like ancestor. Nevertheless, additional biochemical, genetic, phylogenetic and ultrastructural data will be essential in order to reject this null hypothesis and, until that time, the phylogenetic position of Cryptosporidium within the Apicomplexa will remain unresolved.

While this paper was under revision, further support for the early emergence of C. parvum has been provided by an analysis of three genera of the class Gregarina (Carreno et al., 1999). Using only SSU rRNA, these authors have shown that gregarines form a monophyletic clade that is a sister group to Cryptosporidium species and that these genera diverge relatively early from other taxa among the phylum Apicomplexa, including the coccidia, adelids, piroplasms and haemosporinids. These data are congruent with our phylogenetic reconstructions based upon LSU/SSU rRNA and six proteins, as well as SSU rRNA, which support the early emergence of $C$. parvum within the phylum.

\section{ACKNOWLEDGEMENTS}

At Kansas State University, we thank Dr N. V. Nesterenko for the KSU-1 strain oocysts and Dr N. Khramtsov for the pBluescript SK $(+)$ HindIII genomic DNA library; and at the Wadsworth Center we thank both the molecular core 
facility for oligonucleotide synthesis/automated DNA sequencing and the technical assistance of M. J. Marchewka. This research was supported in part by an NCDDG-OI NIH NIAID grant AI-40320 to J.S.K. and a grant from Pharmacia/Upjohn Inc. to J.S.K. and G.Z. All sequences used for alignments and their accession numbers are available both in the EMBL, GenBank or DDJB databases and from the authors upon request.

\section{REFERENCES}

Adachi, J. \& Hasegawa, M. (1996). MOLPHY version 2.3 : programs for molecular phylogenetics based on maximum likelihood. Comput Sci Monogr 28, 1-150.

Barta, J. R., Martin, D. S., Liberator, P. A. \& 9 other authors (1997). Phylogenetic relationships among eight Eimeria species infecting domestic fowl inferred using complete small subunit ribosomal DNA sequences. J Parasitol 83, 262-271.

Bremer, K. (1988). The limits of amino acid sequences data in angiosperm phylogenetic reconstruction. Evolution 42, 795-803.

Brinkmann, H. \& Philippe, H. (1999). Archaea sister group of Bacteria? Indications from tree reconstruction artifacts in ancient phylogenies. Mol Biol Evol 16, 817-825.

Carreno, R. A., Martin, D. S. \& Barta, J. R. (1999). Cryptosporidium is more closely related to the gregarines than to coccidia as shown by phylogenetic analysis of apicomplexan parasites inferred using small-subunit ribosomal RNA gene sequences. Parasitol Res 85, 899-904.

Coombs, G. H. (1999). Biochemical peculiarities and drug targets in Cryptosporidium parvum: lessons from other coccidian parasites. Parasitol Today 15, 333-338.

Coombs, G. H., Denton, H., Brown, S. M. \& Thong, K. W. (1997). Biochemistry of the coccidia. Adv Parasitol 39, 141-226.

Denton, H., Brown, S. M., Roberts, C. W., Alexander, J., McDonald, V., Thong, K. W. \& Coombs, G. H. (1996). Comparison of the phosphofructokinase and pyruvate kinase activities of Cryptosporidium parvum, Eimeria tenella and Toxoplasma gondii. Mol Biochem Parasitol 76, 23-29.

Escalante, A. A. \& Ayala, F. J. (1995). Evolutionary origin of Plasmodium and other Apicomplexa based on rRNA genes. Proc Natl Acad Sci USA 92, 5793-5797.

Fayer, R., Speer, C. A. \& Dubey, J. P. (1997). The general biology of Cryptosporidium. In Cryptosporidium and Cryptosporidiosis, pp. 1-42. Edited by R. Fayer. Boca Raton, FL: CRC Press.

Felsenstein, J. (1985). Confidence limits on phylogenies: an approach using the bootstrap. Evolution 40, 783-791.

Fitch, W. M. (1971). The nonidentity of invariable positions in the cytochromes $c$ of different species. Biochem Genet 5, 231-241.

Fitch, W. M. \& Markowitz, E. (1970). An improved method for determining codon variability in a gene and its application to the rate of fixation of mutations in evolution. Biochem Genet 4 , 579-593.

Gagnon, S., Bourbeau, D. \& Levesque, R. C. (1996). Secondary structures and features of the $18 \mathrm{~S}, 5 \cdot 8 \mathrm{~S}$ and $26 \mathrm{~S}$ ribosomal RNAs from the apicomplexan parasite Toxoplasma gondii. Gene 173, 129-135.

Gajadhar, A. A., Marquardt, W. C., Hall, R., Gunderson, J., AriztiaCarmona, E. V. \& Sogin, M. L. (1991). Ribosomal RNA sequences of Sarcocystis muris, Theileria annulata and Crypthecodinium cohnii reveal evolutionary relationships among apicomplexans, dinoflagellates, and ciliates. Mol Biochem Parasitol 45, 147-154.
Goggin, C. L. \& Barker, S. C. (1993). Phylogenetic position of the genus Perkinsus (Protista, Apicomplexa) based on small subunit ribosomal RNA. Mol Biochem Parasitol 60, 65-70.

Graybeal, A. (1998). Is it better to add taxa or characters to a difficult phylogenetic problem? Syst Biol 47, 9-17.

Hendy, M. \& Penny, D. (1989). A framework for the quantitative study of evolutionary trees. Syst Zool 38, 297-309.

Jones, D. T., Taylor, W. R. \& Thornton, J. M. (1992). The rapid generation of mutation data matrices from protein sequences. Comput Appl Biosci 8, 275-282.

Keithly, J. S., Zhu, G., Upton, S. J., Woods, K. M., Martinez, M. P. \& Yarlett, N. (1997). Polyamine biosynthesis in Cryptosporidium parvum and its implications for chemotherapy. Mol Biochem Parasitol 88, 35-42.

Kim, J. (1996). General inconsistency conditions for maximum parsimony: effects of branch lengths and increasing numbers of taxa. Syst Biol 45, 363-374.

Kimura, M. (1980). A simple method for estimating evolutionary rates of base substitutions through comparative studies of nucleotide sequences. J Mol Evol 16, 111-120.

Kishino, H., Miyata, T. \& Hasegawa, M. (1990). Maximum likelihood inference of protein phylogeny, and the origin of chloroplasts. J Mol Evol 31, 151-160.

Kohler, S., Delwiche, C. F., Denny, P. W., Tilney, L. G., Webster, P., Wilson, R. J., Palmer, J. D. \& Roos, D. S. (1997). A plastid of probable green algal origin in apicomplexan parasites. Science 275, 1485-1489.

Lang-Unnasch, N., Reith, M. E., Munholland, J. \& Barta, J. R. (1998). Plastids are widespread and ancient in parasites of the phylum Apicomplexa. Int J Parasitol 28, 1743-1754.

Lecointre, G., Philippe, H., Le, H. L. V. \& Le Guyader, H. (1993). Species sampling has a major impact on phylogenetic inference. Mol Phylogenet Evol 2, 205-224.

Lockhart, P. J., Larkum, A. W., Steel, M., Waddell, P. J. \& Penny, D. (1996). Evolution of chlorophyll and bacteriochlorophyll: the problem of invariant sites in sequence analysis. Proc Natl Acad Sci USA 93, 1930-1934.

Lopez, P., Forterre, P. \& Philippe, H. (1999). The root of the tree of life in the light of the covarion model. J Mol Evol 49, 496-508.

Maddison, W. P. (1997). Gene trees in species trees. Syst Biol 46, 523-536.

Miyamoto, M. M. \& Fitch, W. M. (1995). Testing species phylogenies and phylogenetic methods with congruence. Syst Biol 44, 64-76.

Moreira, D., Le Guyader, H. \& Philippe, H. (1999). Unusually high evolutionary rate of the elongation factor 1 alpha genes from the Ciliophora and its impact on the phylogeny of eukaryotes. Mol Biol Evol 16, 234-245.

Morrison, D. A. \& Ellis, J. T. (1997). Effects of nucleotide sequence alignment on phylogeny estimation: a case study of $18 \mathrm{~S}$ rDNAs of apicomplexa. Mol Biol Evol 14, 428-441.

Mugridge, N. B., Morrison, D. A., Johnson, A. M., Luton, K., Dubey, J. P., Votypka, J. \& Tenter, A. M. (1999). Phylogenetic relationships of the genus Frenkelia: a review of its history and new knowledge gained from comparison of large subunit ribosomal ribonucleic acid gene sequences. Int $J$ Parasitol 29, 957-972.

Philippe, H. (1993). MUST, a computer package of Management Utilities for Sequences and Trees. Nucleic Acids Res 21, $5264-5272$ 
Philippe, H. \& Adoutte, A. (1998). The molecular phylogeny of Eukaryota: solid facts and uncertainties. In Evolutionary Relationships among Protozoa, pp. 25-56. Edited by G. H. Coombs, K. Vickerman, M. A. Sleigh \& A. Warren. London: Kluwer.

Philippe, H. \& Laurent, J. (1998). How good are deep phylogenetic trees? Curr Opin Genet Dev 8, 616-623.

Poe, S. \& Swofford, D. L. (1999). Taxon sampling revisited. Nature 398, 299-300.

Riordan, C. E., Langreth, S. G., Sanchez, L. B., Kayser, O. \& Keithly, J. S. (1999). Preliminary evidence for a mitochondrion in Cryptosporidium parvum: phylogenetic and therapeutic implications. $J$ Eukaryot Microbiol 46, 52S-55S.

Rogers, M. J., Gutell, R. R., Damberger, S. H., Li, J., McConkey, G. A., Waters, A. P. \& McCutchan, T. F. (1996). Structural features of the large subunit rRNA expressed in Plasmodium falciparum sporozoites that distinguish it from the asexually expressed subunit rRNA. RNA 2, 134-145.

Saitou, N. \& Nei, M. (1987). The neighbor-joining method: a new method for reconstructing phylogenetic trees. Mol Biol Evol 4, 406-425.

Siddall, M. E., Reece, K. S., Graves, J. E. \& Burreson, E. M. (1997). 'Total evidence' refutes the inclusion of Perkinsus species in the phylum Apicomplexa. Parasitology 115, 165-176.

Strimmer, K. \& von Haeseler, A. (1996). Quartet puzzling: a quartet maximum-likelihood method for reconstructing tree topologies. Mol Biol Evol 13, 964-969.

Swofford, D. L. (1993). PAUP: phylogenetic analysis using parsimony, version 3.1.1. Champaign: Illinois Natural History Survey.

Tamura, K. \& Nei, M. (1993). Estimation of the number of nucleotide substitutions in the control region of mitochondrial DNA in humans and chimpanzees. Mol Biol Evol 10, 512-526.

Van de Peer, Y. \& De Wachter, R. (1997). Evolutionary relationships among the eukaryotic crown taxa taking into account site-to-site rate variation in 18S rRNA. J Mol Evol 45, 619-630.

Van de Peer, Y., Robbrecht, E., de Hoog, S., Caers, A., De Rijk, P. \& De Wachter, R. (1999). Database on the structure of small subunit ribosomal RNA. Nucleic Acids Res 27, 179-183.

Van der Auwera, G. \& De Wachter, R. (1998). Structure of the large subunit rDNA from a diatom, and comparison between small and large subunit ribosomal RNA for studying stramenopile evolution. J Eukaryot Microbiol 45, 521-527.

Wilson, R. J. \& Williamson, D. H. (1997). Extrachromosomal DNA in the Apicomplexa. Microbiol Mol Biol Rev 61, 1-16.

Woods, K. M., Nesterenko, M. V. \& Upton, S. J. (1996). Efficacy of 101 antimicrobials and other agents on the development of Cryptosporidium parvum in vitro. Ann Trop Med Parasitol 90, 603-615.

Yang, Z. H. (1996). Phylogenetic analysis using parsimony and likelihood methods. J Mol Evol 42, 294-307.

Yang, Z. H. (1997). Phylogenetic analysis by maximum likelihood (PAML), version 1.3. Berkeley, CA: University of California at Berkeley.

Zhu, G. \& Keithly, J. S. (1997). Molecular analysis of a P-type ATPase from Cryptosporidium parvum. Mol Biochem Parasitol 90, 307-316.

Zhu, G., Marchewka, M. J. \& Keithly, J. S. (2000). Cryptosporidium parvum appears to lack a plastid genome. Microbiology 146, 315-321. 